Revue

de /histoire des religions
Revue de l'histoire des religions

$4 \mid 2015$

Figures de Noé de Gilgamesh au Coran

Noé et son caractère axial dans la littérature du Second Temple

Noah as an Axial Character in the Second Temple Literature

Mădălina Vârtejanu-Joubert

\title{
OpenEdition
}

Édition électronique

URL : http://journals.openedition.org/rhr/8466

DOI : $10.4000 /$ rhr.8466

ISSN : 2105-2573

Éditeur

Armand Colin

Édition imprimée

Date de publication : 1 décembre 2015

Pagination : $519-543$

ISBN : 978-2-200-93012-7

ISSN : 0035-1423

Référence électronique

Mădălina Vârtejanu-Joubert, " Noé et son caractère axial dans la littérature du Second Temple », Revue de l'histoire des religions [En ligne], 4 | 2015, mis en ligne le 01 décembre 2018, consulté le 24 avril 2019. URL : http://journals.openedition.org/rhr/8466 ; DOI : 10.4000/rhr.8466 


\section{Noé et son caractère axial dans la littérature du Second Temple}

Un certain nombre de textes majeurs écrits ou circulant à l'époque du Second Temple - 1 Hénoch, Jubilés, Apocryphe de la Genèse et quelques autres fragments découverts à Qumrân-accordent une place significative au personnage de Noé, à sa vie et à sa geste. Noé garde, comme dans le livre de la Genèse, le caractère de personnage "axial » qui marque un "avant » et un "après » dans l'histoire de l'humanité, mais les fonctions qui lui sont assignées sont différentes. Il est notamment présenté comme prêtre et exorciste, comme dépositaire d'un savoir essentiel transmis à l'aide d'un livre et comme celui qui, après le déluge, partage la terre entre les fils de Sem, Cham et Japhet.

\section{Noah as an Axial Character in the Second Temple Literature}

Some important works such as 1 Henoch, Jubilees, Genesis Apocryphon and other short Qumran fragments, composed or circulating during the Second Temple period, show much interest in the character of Noah and in his life and deeds. He maintains the same "axial" position as in the book of Genesis but his concrete functions are different. Noah is depicted as a priest and exorcist, as the depositary of an essential knowledge enclosed in a book, and as the divider of the Land between his sons Sem, Ham and Japhet. 


\section{INTRODUCTION}

À l'époque du Second Temple et particulièrement entre le $\mathrm{III}^{\mathrm{e}}$ et le $\mathrm{I}^{\text {er }}$ siècle av. n. è., la figure de Noé connaît un riche développement littéraire. Un certain nombre de textes majeurs écrits ou circulant à cette époque - 1 Hénoch, Apocryphe de la Genèse (ou Histoire des Patriarches), Jubilés et, marginalement, quelques autres fragments découverts à Qumrân - accordent une place significative au personnage de Noé, à sa vie et à sa geste. Pour autant, ces textes forment-ils un corpus, autrement dit répondent-ils d'emblée à un critère en vertu duquel ils doivent être étudiés ensemble ? Un premier écueil à éviter est l'appellation «textes non-canoniques» car, pour le propos qui nous concerne ici, elle introduit une distinction anachronique : la question du canon n'est pas tranchée aux $\mathrm{III}^{\mathrm{e}-}$ $\mathrm{II}^{\mathrm{e}}$ siècles av. n. è., date présumée de la composition de ces textes. Pour des raisons similaires - anachronisme et vision téléologique de l'histoire - leur désignation en tant que « textes intertestamentaires », catégorie historiographique souvent utilisée, est également inappropriée ${ }^{1}$. Parmi ces textes, certains, comme l'Apocryphe de la Genèse, furent découverts uniquement à Qumrân; d'autres, comme 1 Hénoch et Jubilés, sont attestés de manière fragmentaire à Qumrân mais sont connus surtout en version éthiopienne. Par ailleurs, les fragments qumrâniens des Jubilés et d'Hénoch ne coïncident pas avec les passages correspondant à l'épisode de Noé. Enfin, ils sont probablement tous des écrits pré-communautaires dont la composition précède la constitution du Yahad.

Ce qui nous autorise cependant à les aborder ensemble ce sont les ressemblances de contenu. Ce constat a donné lieu à une riche bibliographie autour de la question des emprunts et de la filiation littéraire entre ces documents. La manière de représenter la figure de Noé s'inscrit dans cette même logique : d'une part elle obéit à une certaine cohérence de traitement à travers notre corpus et, d'autre part, elle se distingue de l'image qui se dégage des

1. On pourrait ajouter le fait que le terme «intertestamentaire» suppose une période chronologique comprise entre l'Ancien et le Nouveau Testament, ce qui ne correspond pas à la réalité historique : le canon de l'Ancien Testament n'est pas entièrement scellé avant celui du Nouveau Testament. 
passages concernant cette figure dans le livre de la Genèse. Noé garde, certes, le caractère de personnage « axial $»^{2}$ qui marque un « avant» et un «après » dans l'histoire de l'humanité, mais les fonctions qui lui sont assignées sont différentes. Il est notamment présenté comme prêtre et exorciste, comme dépositaire d'un savoir essentiel transmis à l'aide d'un livre et comme celui qui, après le déluge, partage la terre entre les fils de Sem, Cham et Japhet.

Avant de discuter chacune de ces fonctions, soulignons le défi théorico-méthodologique soulevé par l'analyse de ce corpus. Comme nous l'avons indiqué, le Noé de ces textes est différent du Noé de la Genèse. Cette différence est-elle à interpréter comme le résultat d'une exégèse sur un texte faisant autorité - la Genèse - ou, au contraire, comme un processus autonome de transformation et variation d'un mythe, sans postuler un texte de base ? Faudrait-il s'appuyer sur le personnage appelé Noé ou prendre en considération la fonction médiatrice et salvatrice d'un tel personnage, indépendamment de son nom ? Le déplacement d'une telle fonction de Noé vers un autre personnage - son frère par exemple - est-il révélateur d'une polémique anti-noachique ou s'inscrit-il dans la logique implacable de la transformation du mythe ? La question du canon et la question du mythe dans le judaïsme demeurent irrésolues, suscitant chez l'historien un sentiment de grande frustration puisque toute contextualisation demeure hautement incertaine.

Quels que soient les textes du judaïsme antique, Noé y figure en tant que réparateur situé à l'articulation entre un monde ancien, hautement corrompu, et un monde renouvelé, imparfait mais définitif. En deçà de cette généricité, les détails foisonnent : l'origine de la corruption, les modalités de réparation, la nature du nouveau monde, les conséquences à long terme (comme préfiguration du particularisme juif) sont autant d'éléments qui subissent des variations considérables et porteuses de sens.

2. Nous faisons nôtre le terme de personnage «axial», employé par Michael Stone dans un article important sur l'axe de l'histoire à Qumrân : "The Axis of History at Qumran », dans Esther G. Chazon, Michael E. Stone (éds), Pseudepigraphical Perspectives: The Apocrypha and the Pseudepigrapha in Light of the Dead Sea Scrolls. Proceedings of the Second International Symposium of the Orion Center for the Study of the Dead Sea Scrolls and Associated Literature, 12-14 January 1997, Leiden, Brill, 1998, pp. 133-150. 
La version préservée dans le livre de la Genèse, nous présente un personnage de Noé sommairement esquissé, suivant les canons de l'art narratif biblique ${ }^{3}$ : absence de détails, absence de discours direct, de dialogue ou d'introspection, en somme un sujet-agi et non pas un sujet-acteur. Sa naissance, décrite en termes miraculeux dans notre corpus, est totalement passée sous silence dans la Genèse qui préfère introduire le personnage par le biais d'un midrach donnant un sens à son nom : $n w h$, « repos » est dérivé ici de la racine $n h m$, « consoler» (Gn 5.29). L'origine du mal sur la terre est décrite en des termes ambigus dans un passage qui conserve les traces d'harmonisation rédactionnelle. L'union sexuelle entre les elilim (fils des dieux ?) et les filles des hommes semble avoir provoqué la corruption sur terre mais le lien n'est pas explicite. De la même manière, on ignore pourquoi « la méchanceté de l'homme ( $\left.r a^{\prime} a t ~ h a-a d a m\right)$ était grande sur la terre et chaque jour son cœur ne concevait que des pensées mauvaises $\left(\mathrm{ra}^{\circ}\right) »(\mathrm{Gn} 6.5)$. Ou, plus loin, « la terre était corrompue et pleine de violence » (Gn 6.11) et Dieu décida de la détruire ainsi que tout être vivant (kol basar). Mais l'intransigeance divine se trouve tempérée par l'existence d'un être d'exception : « Noé trouva grâce aux yeux du Seigneur » (Gn 6.8) et « Noé était un homme juste (tsaddiq) intègre (tamim) parmi ses contemporains ; il marchait avec Dieu » (Gn 6.9). Noé est chargé d'assurer la survie du monde créé en recueillant auprès de lui, dans l'arche dont Dieu lui ordonne la construction, un exemplaire de chaque couple d'êtres vivants. La fonction du déluge qui s'abat sur la terre et qui s'arrête parce que «Dieu se souvint de Noé », n'est pas claire : est-il destiné à détruire les êtres vivants ou à purifier la terre de la méchanceté de l'homme ? Enfin, le seul geste de Noé que ce texte n'attribue pas à un commandement divin est la construction d'un autel et le sacrifice de tout animal pur. Le sujet agi devient ici joueur de tours, rusé et vrai médiateur car l'odeur agréable des offrandes animales persuade Dieu de ne plus «maudire le sol à

3. À ce titre Hermann Gunkel (notamment Genesis, commentaire paru en trois éditions dont la dernière à Göttingen, Vandenhoeck \& Ruprecht, 1910) reste toujours une référence dont les conclusions sont reprises successivement dans les synthèses consacrées à l'art narratif dans la Bible. Voir la plus récente qui, traduite en une langue de circulation, s'imposerait comme une référence : Frank Polak, Biblical Narrative : Art and Design, Mossad Bialik, Jérusalem, 1994 (en hébreu Ha-Sippour ba-Miqra). 
cause de l'homme», ni de «frapper tout ce qui est vivant». Tout au contraire, Dieu établit une alliance avec Noé, ses fils et leur descendance ainsi qu'avec les êtres vivants qui les accompagnent, stipulant l'interdit du sang, l'obligation pour l'homme de « croître et se multiplier » (Gn 9.7) et l'irréversibilité de la création. Le signe de cette alliance est dorénavant l'arc-en-ciel qui rappellera à Dieu l'engagement de ne plus transformer les eaux des pluies en déluge: une nouvelle limite est posée. C'est ici qu'on peut situer la fin du récit noachique de la Genèse, en notant que l'épisode du partage de la terre entre Sem, Cham et Japhet, ne figure pas dans cette version. En revanche, toutes les autres caractéristiques de Noé relevées dans ce bref résumé, trouvent un écho plus ou moins prononcé dans le corpus littéraire du Second Temple retenu ici, ce qui rend difficile de savoir si nous sommes en présence d'une réécriture exégétique de la Bible ou d'un ensemble de variantes parallèles.

Dans notre article, nous nous proposons d'analyser les multiples facettes du personnage de Noé telles qu'elles se déploient dans trois œuvres majeures de l'époque du Second Temple, 1 Hénoch, Jubilés et Apocryphe de la Genèse. Nous laisserons de côté les nombreux fragments qumrâniens qui, sans mentionner explicitement son nom, peuvent, par association d'idées, être considérés comme faisant partie d'une «tradition noachique». Nous considérons que ces fragments n'apportent rien de plus à la discussion de fond sur les fonctions de Noé et son statut dans le dispositif de pensée du judaïsme de cette époque ${ }^{4}$. La littérature secondaire portant sur la figure de Noé et comprenant à la fois des études qui lui sont spécialement consacrées et des commentaires généraux sur 1 Hénoch, Jubilés et Apocryphe de la Genèse, est très riche ${ }^{5}$. Cette

4. À ce sujet on peut consulter Dorothy M. Peters, Noah Traditions in the Dead Sea Scrolls : Conversations and Controversies of Antiquity, Leiden, Brill, 2008.

5. M. Bernstein, «Noah and the Flood at Qumran», dans D.W. Parry, E. Ulrich (éds), The Provo International Conference on the Dead Sea Scrolls : Technological Innovations, New Texts, and Reformulated Issues, Leiden, Brill, 1999, pp. 199-231; D. Dimant, « Noah in Early Jewish Literature», dans M.E. Stone, T.A. Bergren (éds), Biblical Figures Outside the Bible, Harrisburg, Trinity Press International, 1998, pp. 123-150 ; F. García Martínez, Qumran and Apocalyptic, Leiden, Brill, 1992, pp. 24-44 ; F. García Martínez, «Interpretation of the Flood in the Dead Sea Scrolls », dans F. García Martínez, G.P. Luttikhuizen (éds), Interpretations of the Flood, Leiden, Brill, 1998, pp. 86-108 ; J. Lewis, A Study of the Interpretation of Noah and the Flood in Jewish and Christian Literature, Leiden, Brill, 1968 ; J. VanderKam, « The Righteousness of Noah », 
bibliographie est consacrée, dans sa très grande majorité, à des aspects d'histoire littéraire, à la publication de manuscrits et à des questions philologiques restreintes. Le présent article ne pouvait ni se limiter à explorer des aspects ponctuels, ni juste produire une «vue de haut» sur le Noé du Second Temple. Autant que cela puisse paraître étonnant, il manquait encore une analyse suivie de la façon dont chacun de ces textes élabore la figure de Noé. Par une telle analyse, notre article met en évidence des variations importantes. Mais notre approche ne se situe pas uniquement sur le terrain de l'analyse littéraire, elle prend le parti d'une lecture en clé mythologique. Une application du modèle lévi-straussien sur la variation et la transformation des mythes était prématurée mais le questionnement s'inspire clairement de cet auteur. Nous cherchons à mettre en évidence la signification du personnage de Noé à la lumière de la fonction qu'il accomplit dans une économie mythologique. Comme nous le verrons, cette démarche met en lumière des interrogations de portée philosophique très importante, comme par exemple celle de l'origine épistémique et sexuelle du mal et celle de la réparation du mal dans le monde. Cet article se veut donc, un premier pas dans une réflexion sur ces questions qui, paradoxalement encore, attendent leur traitement systématique pour ce qui est de la littérature juive du Second Temple.

Pour présenter et se pencher sur chaque texte, nous allons suivre le consensus scientifique en matière de datation et d'ordre chronologique, tout en précisant que ce consensus est constamment remis en cause. De l'aveu même des chercheurs, les arguments en faveur d'une certaine datation ou généalogie littéraire ne sont pas irrévocables. En prenant comme appui la datation des fragments découverts à Qumrân, la succession chronologique qui semble emporter le consensus est la suivante : 1 Hénoch (environ 200 av. n. è. ou avant) suivi de Jubilés (environ 160 av. n. è.). Dans la même

dans J. J. Collins, G.W.E. Nickelsburg (éds), Ideal Figures in Ancient Judaism : Profiles and Paradigms, Chico, Scholars Press, 1980, pp. 13-32 ; J. VanderKam, "The Birth of Noah », dans Z.J. Kapera (éd.) Intertestamental Essays in Honor of Jósef Tadeusz Milik, Cracovie, The Enigma Press, 1992, pp. 213-231 ; Cana Werman, "Qumran and the Book of Noah », dans E. Chazon, M. E. Stone (éds), Pseudepigraphic Perspectives: The Apocrypha and the Pseudepigrapha in Light of the Dead Sea Scrolls, Leiden, Brill, 1999, pp. 171-181 ; Michael E. Stone, Aryeh Amihay, Vered Hillel (éds), Noah and his book(s), Atlanta, Society of Biblical Literature, 2010. 
fourchette, et dans un rapport indéterminé avec les autres écrits, se situe l'Apocryphe de la Genèse ${ }^{6}$.

Comme nous l'avons souligné, le personnage de Noé se dévoile à travers les trois séquences narratives qui caractérisent le récit de l'axialité : l'avant, autrement dit la dégradation du monde et l'origine du mal ; la destruction du monde et ses accents eschatologiques ; l'après, autrement dit la guérison du monde par le sacrifice et/ou l'exorcisme dont Noé possède et transmet le savoir et l'organisation du monde nouveau.

\section{HÉNOCH}

Dans le recueil connu aujourd'hui sous le titre de 1 Hénoch $^{7}$ et qui regroupe plusieurs sections distinctes, Noé figure, de manière explicite ou implicite, dans plusieurs passages : dans le Livre des Veilleurs (1 Hén VI-XI), dans le Livre des Paraboles (1 Hén LIV.7-LV.2, 1 Hén LXV.1-LXVIII.1) et dans la dernière section dite des Parénèses (1 Hén CVI-CVII).

Le Livre des Veilleurs commence par l'épisode de la descente des anges sur terre et de leurs méfaits sous la conduite de Shemêhaza et Azaël ${ }^{8}$. Cet épisode est suivi de l'annonce faite au «fils de Lamech» du châtiment des anges et du salut des justes ainsi que

6. Voir la récente discussion et la bibliographie dans Daniel Machiela, The Dead Sea Genesis Apocryphon : A New Text and Translation With Introduction and Special Treatment of Columns 13-17, Leiden, Brill, 2009.

7. Les principaux commentaires sont G.W.E Nickelsburg, 1 Enoch : A Commentary on the Book of 1 Enoch, Chapters 1-36 ; 81-106, Minneapolis, 2001 ; M. Black, The Book of Enoch or 1 Enoch : A New English Edition with Commentary and Textual Notes in Consultation with James VanderKam, Leiden, Brill, 1985; Loren T. Stuckenbruck, 1 Enoch 91-108, Berlin-New York, Walter de Gruyter, 2007.

Pour les citations nous avons suivi A. Caquot, «1 Hénoch », dans A. DupontSommer, M. Philonenko (éds), La Bible. Les Ecrits intertestamentaires, Gallimard, Paris, 1985, pp. 463-625.

Pour les fragments découverts à Qumrân, voir J. T. Millik, The Book of Enoch. Aramaic Fragments of Qumran Cave 4, Oxford, Clarendon Press, 1976, M. Langlois, Le premier manuscrit du Livre d'Hénoch. Etude épigraphique et philologique des fragments araméens 4Q201 à Qumrân, Cerf, Paris, 2008.

Voir aussi A. Yoshiko Reed, Fallen Angels and the History of Judaism and Christianity. The Reception of Enochic Literature, Cambridge, 2005.

8. Pour une mise au point d'histoire littéraire voir Mathias Delcor, « Le mythe de la chute des anges et de l'origine des géants comme explication du mal dans le monde », RHR, 1976, pp. 3-53. 
par l'intercession infructueuse d'Hénoch en faveur des anges déchus et des géants. Pour notre propos, ces passages illustrent le premier volet du récit axial, à savoir la description d'un état du monde qui nécessite réparation. Dans ces extraits de 1 Hénoch, l'origine du mal n'incombe pas directement aux hommes mais aux anges, responsables d'avoir incité l'humanité aux péchés. Le texte, comme celui de la Genèse, n'est pas exempt d'ambiguïtés. C'est d'abord l'attraction physique envers les «filles des hommes » qui conduit les «fils du ciel » à commettre un «grand péché » (1 Hén VI.1-49). Quelques phrases plus loin, on apprend cependant que le péché ne se ramène pas uniquement à une union corporelle contre-nature (entre un être humain et un être céleste) et de ce fait entachée de souillure. Le péché consiste aussi, et peut-être surtout, dans la révélation de savoirs considérés comme étant des mystères célestes : les drogues, les charmes, la botanique, la métallurgie, le fard à paupières, les pierres précieuses et les couleurs, les exorcismes, la magie et les tours, l'astrologie, la science des signes (1 Hén VII.1, VIII.1-3). Quelle en fut la conséquence?

D'une part, cette union hybride donna naissance à des êtres hors normes, «des géants hauts de trois mille coudées » qui agissent immanquablement à l'encontre de l'ordre établi :

Ils dévorèrent tout le fruit du labeur des hommes, si bien que les hommes ne purent plus les nourrir. Les géants se liguèrent contre eux pour les tuer et dévorèrent les hommes. Ils se mirent à pécher contre toutes (les betes), oiseaux, quadrupèdes, reptiles, poissons, et à se dévorer entre eux. Ils burent du sang. Alors la terre accusa les criminels pour ce qui y avait été fait. (1 Hén VII.3-4).

D'autre part, de l'enseignement des savoirs mystérieux :

Il en résulta une grande impiété. (Les hommes) se débauchèrent, s'égarèrent et se perdirent dans toutes leurs voies. (1 Hén VIII.2)

Ces deux thèmes sont juxtaposés dans 1 Hén IX.4-11 laissant entrevoir en filigrane, l'idée d'une consubstantialité civilisatrice

9. « ont abandonné les hauteurs célestes, le sanctuaire de la résidence éternelle, pour se souiller au contact des femmes, et qui agissent comme les fils de la terre et ont pris femme. [E. Ils ont apporté] à la terre une grande désolation, et il n'y aura pour [eux] ni paix ni rémission. » (1 Hén XII.4-5). Pour le jugement définitif des anges voir 1 Hén XIV.24-XV.4. - Sur l'union des fils de Dieu avec les filles des hommes et la révélation des mystères divins voir Valérie Triplet-Hitoto, Mystères et connaissances cachées à Qumrân, Cerf, Paris, 2011, pp. 53 ss et passim (avec bibliographie). 
entre l'érotisme et la connaissance ${ }^{10}$. Cette idée s'enracine sans doute dans la polysémie du verbe hébreu yada ' qui signifie à la fois « connaître » et « accomplir l'acte sexuel », puisque l'hébreu forme le substrat linguistique et conceptuel de ce livre et des traductions qui nous sont parvenues.

L'intimité physique des anges et des femmes conduit donc à une intimité épistémique qui entraîne à son tour un désordre généralisé qualifié de " péché » et de "violence ». Alors que dans Genèse 6 Dieu constate par lui-même la méchanceté instaurée parmi les humains, dans 1 Hénoch ce sont les humains qui ressentent le désordre comme tel et « crient vers Dieu » pour demander de l'aide. Commence ainsi la deuxième étape du récit axial, l'intervention divine qui, dans ces passages, se manifeste avec une grande complexité. La figure du médiateur s'habille de contours beaucoup plus flous que dans le livre de la Genèse. Le passage qui pourrait se rapporter à Noé, désigné non pas par son nom mais par son ascendance en tant que « fils de Lamech », tient ici en trois phrases par lesquelles l'ange Ouriel est chargé de lui révéler l'avenir de la terre et des hommes :

Ordonne-lui en Mon nom de se cacher et annonce-lui que la fin est proche : toute la terre va périr, un déluge va arriver sur toute la terre et détruire tout ce qu'elle porte. Enseigne-lui le moyen d'y échapper, et sa semence subsistera dans toutes les générations du monde. (1 Hén X.1-3)

Le nom du personnage n'est pas ici digne de mention alors qu'il suscite une étymologie dans la Genèse. Ses mérites et sa fonction ne sont pas explicités ; la construction de l'arche est absente. Nous retenons l'imminence du déluge dont la description est elle-aussi implicite. Le sort de la terre se lit à travers le sort infligé aux anges coupables :

le jour du grand Jugement il (Azaël) sera conduit dans la fournaise. La terre que les anges ont souillée sera assainie. (Raphaël) Annonce la guérison de la terre : on guérira sa plaie, et tous les humains ne périront pas à cause de tout le mystère meurtrier que les Veilleurs ont enseigné à leurs fils. (1 Hén X. 6-7)

Dans cette version, l'axialité de Noé est très effacée car non seulement il n'intervient pas dans la réparation du monde mais il

10. A. Caquot note à cet endroit la «tendance encratite de l'auteur. » Op. cit., p. 478, note sur 1 Hén VII.1. Comme indiqué dans le corps de l'article, notre interprétation est différente. 
n'est pas non plus le seul juste sauvé, ancêtre de toute la nouvelle humanité. Ce mythe met l'accent sur la continuité du monde pardelà le jugement des anges pécheurs. Dans l'adresse à l'ange exterminateur Michel, Dieu ordonne :

Fais périr tous les esprits des dépravés et les fils des Veilleurs, parce qu'ils ont exercé la violence contre les hommes. Fais disparaître toute la violence de la surface de la terre; que cesse toute œuvre de perversité ; que la plantation de justice et de vérité apparaisse [E et ce sera une bénédiction ; les œuvres de justice et de vérité seront pour l'éternité] plantées dans la joie. (1 Hén X.15-16)

Le troisième acte du récit axial, la réparation, se lit à travers le sort des justes :

Et maintenant, tous les justes vont échapper, ils resteront en vie jusqu'à ce qu'ils aient engendré des milliers (de descendants). Tous les jours de leur jeunesse et de leur vieillesse s'accompliront dans la paix. Alors toute la terre sera travaillée dans la justice, il y sera planté un arbre, et elle sera comblée de bénédiction. Tous les arbres de la terre seront plantés; on y plantera des vignes, et toute vigne plantée produira des jarres de vin par milliers; chaque mesure de grain en produira [mille, une seule mesure] d'olives produira dix baths [d'huile]. (1 Hén X.17-19)

Dans ce passage, comme dans le suivant, le lecteur est tenté de voir plutôt le moment eschatologique que la coupure médiane correspondant au déluge. La pacification de la terre et la fertilité exponentielle sont des motifs messianiques connus ${ }^{11}$. Mais malgré les allusions intertextuelles à l'époque messianique, ces passages ne renvoient pas explicitement à la fin des temps. Structurellement, le motif de la fertilité qui succède au cataclysme réparateur est présent aussi dans la Genèse 8.22 ; 9.1. De même, le motif de la purification de la terre semble lui-aussi structurel à ce récit même si l'expression textuelle n'en est pas la même : dans la Genèse Noé bâtit un autel et offre un sacrifice (8.20), tandis que dans 1 Hénoch l'ange Michel doit procéder à la purification de la terre et des péchés sans en préciser les détails.

Le récit se termine par un corollaire universaliste, ce qui accentue la ressemblance avec la période correspondant à la venue du Messie :

[E Tous les hommes seront justes,] tous les peuples Me serviront, tous ils Me béniront et M'adoreront. La terre entière sera purifiée de toute souillure, de toute impureté, de toute fureur, de tout châtiment,

11. Voir Amos 9.13-14, Ezéchiel XXVIII.26, 2 Baruch 19.5, Psaume 72. 
et Je ne leur enverrai plus [E de déluge] dans toutes les générations du monde. Alors J'ouvrirai les trésors de bénédiction qui sont dans le ciel, Je les déverserai sur les travaux, sur le labeur des humains. Alors la vérité et la paix s'associeront pour tous les jours du monde et toutes les générations humaines. (1 Hén X.21-XI.2)

La figure de Noé se présente ici sous des traits très vagues, elle est presque absente. Le récit axial tient sa consistance de l'intervention des anges dans le monde : origine du mal et figures expiatoires de la violence engendrée par l'indistinction entre les êtres humains et les êtres divins. La descendance de Noé n'occupe pas la place prééminente que lui réserve la Bible et d'autres passages apocryphes puisque ce n'est pas lui seul qui assure le lien entre la première humanité et la seconde : c'est l'ensemble des justes qui par son salut incarne ce lien. La théodicée attestée dans ce passage se retrouve également dans le Livre des paraboles et dans les Parénèses ainsi que dans le livre des Jubilés et dans l'Apocryphe de la Genèse où Noé devient acteur, prend la parole et joue pleinement son rôle de personnage axial, de chaînon unissant la création et la re-création du monde.

La deuxième section de 1 Hénoch, connue sous le nom de Livre des paraboles, retient notre attention par deux passages de taille et de nature différentes mais qui éclairent la discussion autour de la figure noachique. Le premier, 1 Hén LIV.7-LV.2, contient une description du déluge, le deuxième, 1 Hén LXV.1-LXVIII.1, comporte un long discours de Noé, rompant ainsi avec le laconisme précédent.

La description du déluge dans la deuxième parabole, au chapitre LIV, intervient dans le même contexte que dans le Livre des Veilleurs étudié précédemment, à savoir le châtiment des anges déchus "parce qu'ils ont été au service de Satan et ont égaré les habitants de l'aride. »(1 Hén LIV.6). Le déluge représente ici l'interlude avant le jugement final et le nom de Noé ou de tout autre médiateur est absent. Dieu seul se repent et met un terme à la destruction par les eaux des humains mais aussi de ceux qui habitent « aux franges du ciel » : un signe dans les cieux indiquera la fidélité à la promesse de ne plus traiter de la sorte les habitants de l'aride. La parabole se poursuit ensuite avec le châtiment eschatologique.

La troisième parabole en revanche, fait de Noé une figure centrale puisqu'il prend la parole, prie et se lamente pour le sort de l'aride 
(la terre). Noé commence par faire le constat du « chancellement de la terre » et du fait que «sa destruction était proche» mais n'en saisit pas la cause. Voulant comprendre les raisons du désastre qui s'annonce, il interroge son aïeul, Hénoch :

Raconte-moi ce qui se passe sur la terre pour qu'elle peine et vacille de la sorte. Ne vais-je pas moi aussi périr avec elle ? (1 Hén LXV.3)

Ici, comme précédemment, c'est la connaissance des mystères par les hommes qui est source de châtiment et de destruction. Si le mythe de la Genèse fait de la connaissance du bien et du mal un fruit interdit, le livre d'Hénoch déplace la palette des savoirs ésotériques réservés aux anges de l'éthique vers l'alchimie :

la violence et la puissance des Satans, le mystère des mystères, toute la puissance des sorciers, la puissance des sortilèges, la puissance de ceux qui fondent le métal de toute la terre.

L'incrimination continue en indiquant avec clarté la maîtrise de la métallurgie :

(Ils ont appris) comment l'argent naît de la poussière de la terre et comment le métal fusible est produit sur la terre. Car le plomb et l'étain ne naissent pas de la terre comme le premier (métal) ; c'est une source qui leur donne naissance, un ange s'y tient, et cet ange éparpille (le minerai). (1 Hén LXV.8)

Ce passage est l'expression de l'antique tradition alchimiste dont l'objectif, la transmutation des métaux et la panacée, repose sur le principe de la correspondance des substances, la sympathie universelle. Microcosme et macrocosme sont ainsi liés selon des règles précises associant métaux, couleurs, planètes et, par conséquent, périodes du calendrier. C'est cette tournure que prend la réponse d'Hénoch à Noé :

C'est à cause de leur méfait que leur condamnation s'est accomplie. Ils ne seront plus comptés devant moi à cause des mois qu'ils ont cherché à connaître. La terre périra avec ses habitants. (1 Hén LXV.9-10)

L'innocence de Noé est à comprendre dans ce contexte, dans les deux sens du terme, comme pureté et ignorance à la fois. Son aïeul lui fait savoir que lui seul parmi les hommes sera sauvé car «le Seigneur des Esprits sait que tu es vertueux et pur de cet opprobre venu des mystères ». Noé est aussi préservé parmi «les saints » terme dont l'exégèse s'avère difficile et qui, selon notre avis, renvoie à la future élection d'Israël. Ce ne serait pas l'unique passage où 
Noé est envisagé comme un proto-Abraham ou un proto-Moïse. La promesse faite à Noé ici rappelle fortement celle faite à Abraham en Genèse 17.4ss :

Il a maintenu ta descendance légitime pour (en faire) des rois et pour de grands honneurs, et de ta descendance sortira une source de justes et de saints innombrables, pour l'éternité. (1 Hén LXV.12)

Après avoir reçu ces révélations par l'intermédiaire d'Hénoch, c'est Dieu même qui s'adresse à Noé pour lui annoncer son salut. Dans cette deuxième prophétie le style et le vocabulaire changent et il est probable qu'il n'y ait pas de continuité littéraire entre ces deux chapitres. L'élection de Noé est signifiée par un terme cher aux écrits communautaires de Qumrân, à savoir le «lot» : celui de Noé est « exempt d'opprobre, un lot d'amour et de rectitude », réminiscence peut-être de Gn 6.8-9 (« Mais Noé trouva grâce aux yeux de l'Éternel. [...] Noé était un homme juste et intègre dans son temps ; Noé marchait avec Dieu. »). Pour le sauver, ce sont les anges qui vont construire l'arche qui servira d'abri et de laquelle proviendra «une semence de vie». La recréation du monde sera qualitativement différente puisqu'un « changement se produira afin que l'aride ne demeure point nue ». Le nouveau monde demeure à jamais tout comme la descendance de Noé. Celui-ci retrouve pleinement sa stature de personnage axial puisqu'il se perpétue non seulement physiquement, en engendrant une descendance, mais aussi sur le plan de la connaissance puisqu'il transmet tout le savoir antédiluvien recueilli dans un livre :

Après cela, mon aïeul Hénoch a mis pour moi dans un livre l'explication des mystères, ainsi que les visions symboliques qui lui avaient été accordées. Il les a jointes pour moi au livre des Paraboles. » (1 Hén LXVIII.1)

Une fois de plus éros et épistémè sont associés ce qui dénote une réflexion sur les propriétés de l'humain : sa pérennité dépend de la reproduction à la fois corporelle et incorporelle. Pour créer de l'identique ou du semblable, la seule enveloppe physique ne suffit pas mais nécessite une autre composante, à savoir la transmission d'un ensemble de connaissances.

Le motif de l'écrit, que ce soit sous la forme de tablettes célestes ou de livre, réapparaît dans la troisième partie du livre d'Hénoch, les Parénèses. Ainsi, un long passage est consacré à l'avertissement des 
pécheurs qui se rendent coupables, entre autres, de la falsification des Écritures :

« [...] les pécheurs altèrent et récrivent [E les paroles de vérité], ils en changent la plupart, ils mentent et forgent de grandioses fictions, ils rédigent des Écritures en leur nom. Si seulement ils écrivaient en leur nom toutes mes paroles, fidèlement, sans les abolir ni les altérer, mais en rédigeant fidèlement les témoignages que je leur transmets !

Je sais encore un second mystère : les justes, les saints et les sages recevront mes livres pour se réjouir de la vérité. [E Ils recevront les livres,] ils y accorderont foi et s'en réjouiront, et tous les justes jubileront d'y apprendre toutes les voies de la vérité. » (1 Hén CIV.10-13)

Ces parénèses, incitant les justes à espérer et réprimandant les pécheurs, sont suivies du passage décrivant la coupure noachique, annoncée sous la forme d'une prophétie transmise par Hénoch à son fils, Mathusalem, au sujet de son arrière-petit-fils, Noé. Le récit n'est donc pas celui actuel du déluge mais celui de son avènement inéluctable et il prend comme prétexte non pas le constat d'une déchéance généralisée du monde mais un événement privé, l'apparence sur-humaine du nouveau-né de Lamech, de la lignée de Mathusalem et d'Hénoch. Ce petit enfant montre des signes étranges - la blancheur et la rougeur du corps, les yeux brillants et l'aptitude innée à marcher ${ }^{12}$ - qui incitent le père et le grand-père à penser qu'il n'est pas engendré par un humain mais par un ange. La signification de cet événement miraculeux est dégagée par Hénoch qui, tout en rassurant Mathusalem sur la paternité de Lamech, lui dévoile le cours de l'histoire et le rôle axial et salvateur que son fils allait jouer dans ce scénario : il devra s'appeler Noé car il donnera du « repos » à une humanité en proie au péché et bouleversée par le châtiment diluvien. Sur le plan narratif, on note l'inversion chronologique dans le déroulement du récit axial : la naissance du médiateur attire l'attention du lecteur avant les signes faisant état d'une humanité abîmée et vouée à la déchéance. La tension narrative est suspendue puisque l'issue de la crise est d'avance connue : «ce petit enfant qui vient de naître sera épargné, ses trois

12. Le motif du puer senex, le nourrisson sénescent, est abordé par A. Caquot, "Les enfants aux cheveux blancs. Réflexion sur un motif », in Mélanges d'histoire des Religions offerts à Henri-Charles Puech, Paris, 1974, p. 161-172. Pour une approche comparatiste et une critique de Caquot, voir Christine Dumas-Ruengoat, "Vieillards nourrissons et nourrissons sénescents dans la littérature grecque ancienne », Kentron, 18, 2002, pp. 55-68. Elle analyse ce motif à l'intérieur d'une catégorie mythico-narrative plus large, celle de l'adynaton, « la chose impossible ». 
enfants seront sauvés » et "il guérira la terre du fléau qu'elle aura connu » (1 Hen CVI.16). Dans cette perspective, l'histoire n'est pas seulement prédéterminée mais téléologique car le cycle péchéjugement ne s'arrête pas à l'époque du déluge et à la médiation de Noé, il se répète pour aboutir au jugement dernier. L'histoire est inscrite dans les tablettes célestes :

Par la suite, il y aura encore plus d'injustice qu'auparavant sur la terre. [...] les générations iront en empirant, et cela, je l'ai vu, jusqu'au lever des générations de justice. (Alors) le vice et le péché cesseront, l'injustice sera bannie de la terre, et le bien y viendra pour eux. (1 Hen CVI. 19 ; CVII.1)

Paradoxalement, malgré une meilleure élaboration littéraire du héros grâce à sa naissance miraculeuse, Noé demeure ici un personnage passif, dépourvu de parole et d'action. Il s'intègre au dispositif téléologique dans lequel l'humanité évolue sans pour autant que ses qualités ou ses mérites nous soient communiqués.

Par-delà sa diversité, le recueil hénochique nous présente une version du récit axial dans laquelle l'origine du mal incombe aux anges et où le personnage réparateur est généralement passif, instrument d'une mécanique divine établie à l'avance. Dans la plupart des passages examinés, le déluge représente non pas le règlement définitif d'une crise du monde créé mais la préfiguration du jugement eschatologique. La figure noachique est à la fois marquante et dérisoire puisqu'elle assure la continuité de l'humanité mais pas sa pérennité : en effet, le monde postdiluvien ne sera pas exempt de péché et de destruction.

\section{JuBILÉS}

Le livre des Jubilés ${ }^{13}$ nous présente un Noé différent. Plusieurs chapitres sont consacrés à la période comprise entre la naissance

13. Les principaux commentaires sont J. C. VanderKam, The Book of Jubilees, Peeters, Louvain, 1989, K. Berger, Das Buch der Jubiläen, Gütersloh, Mohn, 1981, M. Segal, The Book of Jubilees, Leiden, Brill, 2007, D. Hamidovic, Les traditions du jubilee à Qumrân, Paris, 2007. - Pour les citations nous avons suivi A. Caquot, «Jubilés », dans A. Dupont-Sommer, M. Philonenko (éds), La Bible. Ecrits intertestamentaires, Gallimard, Paris, 1987, pp. 629-810.

Voir aussi H. Najman, Seconding Sinai. The Development of Mosaic Discourse in second Temple Judaism, Leiden, Brill, 2003. 
de Noé (Jub IV.28) et sa mort (Jub X.17). Malgré l'intertextualité évidente par endroits, la caractérisation générale du personnage ainsi que le message véhiculé sont différents de ceux de la littérature hénochique. Le récit est plus long et plus détaillé, les attributs de Noé plus nombreux - à commencer par sa stature sacerdotale -, le rapprochement avec l'alliance abrahamique et mosaïque plus explicite. La tonalité apocalyptique du livre d'Hénoch qui nous présente un Noé vêtu de traits messianiques, est tempérée ici par un Noé prêtre, législateur et scribe.

Considéré généralement comme appartenant au genre littéraire de la «Réécriture biblique », en espèce la réécriture du livre de la Genèse, le livre des Jubilés nous transmet des traditions complémentaires comme celles concernant le nom de la mère de Noé $\left(\right.$ Betenos $\left.^{14}\right)$, le nom et la généalogie de sa femme (Emzara), les dates de naissance de ses trois fils Sem, Cham et Japhet, etc. Le récit axial se voit ainsi doté d'un certain nombre de précisions significatives, comme celle qui inclut les animaux dans la logique du péché. Certes, nous savons que le déluge détruit toute la surface de la terre et que l'arche de Noé sauve un couple de chaque être vivant, mais seul le livre des Jubilés attribue explicitement la responsabilité du péché, non seulement aux anges et aux hommes, mais aussi aux animaux :

La violence s'accrut sur terre et tous (les êtres) de chair corrompirent leur conduite, depuis les hommes jusqu'aux animaux domestiques et sauvages, aux oiseaux et à tout ce qui marche à terre. Tous corrompirent leur conduite et leurs règles de vie et ils commencèrent à se dévorer entre eux. (Jub V.2)

Cette idée selon laquelle l'homme n'est pas le seul produit de la création doué de responsabilité et d'imputabilité transparaît dans le passage annonçant la recomposition du monde :

Il $<$ fera $>$ pour toute son œuvre une création nouvelle et juste, en sorte que (Ses créatures) ne pécheront plus jamais, en aucune de leur nature, mais elles seront justes en tout temps, chacune selon son espèce. » (Jub V.12) [...]

Il prononcera sur chacun sa sentence, sur le grand poisson en raison de sa grandeur, sur le petit poisson en raison de sa petitesse, sur chacun en raison de sa conduite. (Jub V.15)

14. Bat-Enosh dans l'Apocryphe de la Genèse. 
Fait significatif, cette sentence de portée non seulement universaliste mais cosmique, est suivie d'une incise particulariste sur les enfants d'Israël aux sujets desquels

il a été écrit et décrété que s'ils se tournent vers Lui dans la justice, Il laissera de côté toutes leurs transgressions et pardonnera tous leurs péchés. (Jub V.17)

Ce trait, suggéré en filigrane par le livre d' Hénoch, est pleinement exprimé dans les Jubilés. Si le premier donnait préférence au rapprochement structurel entre le déluge et l'eschaton, le second met l'accent sur l'alliance qui succède à la destruction, sur la recomposition du monde, et inscrit clairement le personnage de Noé dans la lignée d'Abraham et de Moïse. La conception de l'histoire reste téléologique, mais l'auteur est plus concerné par les origines et désireux de déceler la cohérence du plan divin dès la création du monde, que par l'inéluctabilité de sa fin. Noé sert ici à légitimer l'existence $a b$ initio d'une constitution du monde conforme à la Torah, que l'humanité n'a fait qu'oublier et redécouvrir. Les révélations qui suivent ne sont pas des nouveautés historiques mais des prises de conscience : Dieu est constant, l'humanité labile. L'auteur des Jubilés veut ainsi expliquer le particularisme juif en remontant le cours du temps et en faisant de Noé le détenteur d'un corpus législatif que le peuple élu seul aura, par la suite, fait sien.

Un des éléments illustrant ce propos est le sacrifice offert par Noé à la sortie de l'arche (Jub VI.1-3), offrande d'expiation « pour toute la terre » minutieusement décrite et composée d'éléments présents de manière disparate dans les Nombres et le Lévitique. Le « jeune bouc » est mentionné lors de l'inauguration du Tabernacle comme « victime pour le péché » (Nb 7.16) mais aussi comme victime sacrificielle destinée, après l'entrée des tribus dans la terre promise, à racheter une faute communautaire $\left(\mathrm{Nb} 15 \cdot 24^{15}\right)$. L'holocauste qui comprend « un taureau, un bélier, une brebis, des boucs, du sel, une tourterelle et un jeune pigeon » est une synthèse des holocaustes décrits dans Lévitique 1. Enfin, la libation du vin au-dessus d'une oblation pétrie à l'huile n'a pas de correspondant biblique. En

15. «Si la faute a été commise sans que la communauté s'en rende compte, c'est elle qui, dans son ensemble, m'offrira un taureau en sacrifice complet dont j'apprécierai la fumée odorante ; elle y joindra les offrandes réglementaires de farine et de vin, ainsi qu'un bouc en sacrifice pour obtenir le pardon. » 
offrant ce sacrifice, Noé se vêtit des traits sacerdotaux. De même, en acceptant l'interdit de sang et en concluant avec Dieu une alliance éternelle, Noé préfigure Moïse. Le texte établit clairement ce lien en faisant suivre l'alliance noachique de l'alliance mosaïque :

C'est pourquoi Il t'a dit à toi aussi, avec les enfants d'Israël, de conclure une alliance en ce mois sur une montagne, avec un serment, et de les asperger de sang, pour tous les termes de l'alliance que le Seigneur a conclue avec eux pour toujours. Cette attestation est écrite à votre intention, pour que vous ne mangiez jamais le sang d'une bête, d'un oiseau ou d'un animal domestique, pendant toute la durée de la terre. (Jub VI.11-12)

La même logique préside à l'institution de la fête des Semaines qui, avant d'être honorée par les fils d'Israël, fut « célébrée au ciel depuis le jour de la création » et par les fils de Noé jusqu'à la mort de celui-ci quand elle tomba dans l'oubli. L'histoire est ainsi rythmée par l'alternance oubli-remémoration des commandements divins : Abraham, Isaac, Jacob et ses fils observent la fête et l'interdit du sang, mais elle est encore oubliée par la génération contemporaine de Moïse qui «la restaure pour eux auprès de cette montagne » (Jub VI.19). À la différence de la fête de Shavuot dont l'archétype réside dans le monde céleste, d'autres fêtes sont instituées par Noé : les débuts du premier, quatrième, septième et dixième mois.

Enfin, Noé reçoit la stature de législateur transmettant à sa descendance «les ordonnances, les commandements et tout ce qu'il connaissait comme loi. »(Jub VII.20). Cela comprend l'injonction «d'accomplir la justice, de couvrir la honte de leur corps, de bénir leur Créateur, d'honorer père et mère, d'aimer chacun son prochain, de se garder de la fornication, de l'impureté et de toute violence », mais aussi l'interdit du sang et les ordonnances concernant les prémices. Cette fois, la modalité de transmission n'est plus l'écrit et donc les tables célestes, mais la chaîne orale : d'Hénoch à Mathusalem, ensuite à Lamech et en enfin à Noé. La littérature juive d'époque hellénistique comporte, en filigrane, une réflexion sur les rapports entre l'oral et l'écrit. Plus tard, les textes rabbiniques nous transmettrons des positions explicites et tranchées, les considérations sur l'écriture s'inscrivant elles-aussi dans une conception particulariste de l'histoire ${ }^{16}$. La dialectique de

16. Nous avons abordé ces questions dans deux articles : «La lettre comme objet : l'écrit entre réification et herméneutique dans le judaïsme rabbinique », 
l'oral et de l'écrit au sujet du savoir transmis par Noé est un sujet pas encore systématiquement étudié. Entre les tables célestes et la chaine de transmission orale, les conséquences ne sont pas les mêmes, l'écrit étant le vecteur d'une généralisation et d'une décontextualisation qui contrarient la tendance au particularisme et au déterminisme ${ }^{17}$.

Le récit axial comporte ici un dernier acte dont l'acteur principal demeure toujours Noé : le partage de la terre entre ses trois fils au moyen du tirage aux sorts (Jub VIII.19ss). À l'intérieur d'une vision déterministe et téléologique de l'histoire où tout est conçu pour aboutir à l'alliance mosaïque, comment comprendre le paradoxe du hasard qui surgit ici ? Francis Schmidt a bien mis en évidence la double acception du tirage aux sorts dans le judaïsme hellénistique : procédé ouvrant à la révélation de la volonté divine, d'une part, et rituel assurant l'équité de la distribution, d'autre part ${ }^{18}$. Dans le livre des Jubilés, Noé est crédité d'une prise de conscience de sa propre prophétie : «Et Noé se réjouit de voir ce territoire échoir à Sem et à ses fils et il se rappela tout ce qu'il avait prophétisé de sa bouche. Il avait dit en effet « Béni soit le Seigneur, Dieu de Sem et que le Seigneur habite dans les demeures de Sem » (Jub VIII.18). Le tirage aux sorts s'inscrit donc dans le dispositif téléologique qui mène de l'universalisme de l'humanité entière au particularisme des descendants de Sem.

Une fois ce procédé accompli, Noé cherche à garantir sa pérennité en faisant prêter serment à ses fils de respecter chacun les frontières

Tsafon, 58, 2009-2010, pp. 15-30 et « Verba manent : Signs, Sounds, and Letters in rabbinic Literature », in Athalya Brenner-Idan (éd.), Discourse, Dialogue, and Debate in the Bible. Essays in Honour of Frank H. Polak, Sheffield Phoenix Press, Sheffield, 2018, pp. 247-257.

17. On comprend ainsi la mise en parallèle de l'écrit et de l'oral ainsi que la capacité de l'écrit de générer de la folie, dans l'épisode de Kaïnam, descendant de Noé (Jub VIII.3-4) : «il découvrit une inscription que des anciens avaient gravée sur le roc. Il lut et copia ce qui y était (inscrit) et s'égara de ce fait : il s'y trouvait la doctrine des Veilleurs, selon laquelle ils pratiquaient la divination par le soleil, la lune et les étoiles dans tous les signes du ciel. Il la mit par écrit mais n'en dit mot, car il craignait qu'on en parle à Noé qui en aurait été irrité contre lui. »

18. Francis Schmidt, «GÔRAL versus PAYÎS : Casting Lots at Qumran and in the Rabbinic Tradition », in Florentino García Martínez, Mladen Popovič (éds), Defining Identities : We, You, and the Other in the Dead Sea Scrolls : Proceedings of the Fifth Meeting of the IOQS in Groningen, Leiden, Brill, 2008, pp. 175-185. 
ainsi établies. Personnage axial, Noé est dans le livre des Jubilés surtout un poseur de limites : morales, temporelles et spatiales. De même que Dieu se limite à son tour en s'engageant à ne plus détruire la création et à mettre un terme à sa colère cosmique. L'auteur doit cependant expliquer l'imperfection de cette deuxième création et le caractère orienté de l'histoire vers le Jugement dernier. Dans ce but, il fait intervenir à nouveau la corruption des démons et la séduction qu'ils exercèrent à nouveau sur les humains, à commencer par les «enfants des fils de Noé » (Jub X.1). Si l'origine du mal ayant conduit au déluge était expliquée elle aussi par les agissements des démons, la persistance du mal dans le monde est expliquée par la décision divine de laisser libre un dixième des esprits et de faire descendre au « lieu de jugement» le reste (Jub X.9). Au thème de l'installation des limites succède ainsi celui de la continuité ou de la consubstantialité entre l'ancien et le nouveau monde. Ce thème est illustré non seulement par la persistance des démons mais aussi par la constitution d'un livre comprenant tout le savoir antédiluvien révélé par les anges à Noé :

Nous (les anges) avons dit à Noé les remèdes de tous les maux (que les démons infligent) ainsi que les séductions, afin qu'il soigne au moyen des plantes de la terre. Noé a inscrit dans un livre tout ce que nous avons enseigné sur toutes les sortes de remèdes, et les esprits mauvais ont été tenus à l'écart des fils de Noé. Celui-ci donna à son fils aîné Sem tout ce qu'il avait écrit, car il l'aimait le plus parmi tous ses fils. (Jub X.12-14)

À travers la métaphore familiale de la préférence paternelle, nous pouvons lire le cheminement du particularisme juif : le savoir noachique ne se transmet désormais que dans une seule branche de la nouvelle humanité, celle de Sem, ancêtre éloigné d'Abraham. C'est à plusieurs reprises que le livre des Jubilés rapproche Noé et Abraham.

Par exemple, lorsqu' Abraham et, curieusement pas Isaac, impose la bénédiction de Jacob :

C'est grâce à sa descendance que mon nom sera béni ainsi que le nom de mes pères : Sem, Noé, Hénoch, Mahalel, Enosh, Seth et Adam. (Jub XIX.24, voir aussi XIX.27)

Ou lorsqu'il enseigne les lois alimentaires à Isaac :

C'est en effet ce que j'ai trouvé écrit dans les livres de mes pères, dans les paroles d'Hénoch et dans celles de Noé. (Jub XXI.10) 
Ou enfin, lorsqu'il prononce sa dernière bénédiction de Jacob :

Que Dieu t'accorde toutes les bénédictions dont il m'a béni et dont Il a béni Noé et Adam. (Jub XXII.13)

Poseur de limites et chaînon de transition, Noé incarne dans le livre des Jubilés ces deux traits complémentaires. L'ordre du monde institué à son époque et, dans une certaine mesure, par lui-même, est conçu comme une préfiguration des règlements de l'époque abrahamique et mosaïque.

\section{Apocryphe de la Genèse}

Le troisième texte que nous considérons comme révélateur des traits de Noé dans la littérature du Second Temple est connu sous le titre d'Apocryphe de la Genèse ou, selon son dernier traducteur, Histoire des Patriarches ${ }^{19}$, et il est attesté uniquement parmi les manuscrits découverts à Qumrân. Le rouleau - désigné par le sigle 1Q20 -, extrêmement endommagé, comporte un texte en araméen considéré lui-aussi comme une « réécriture » des premiers chapitres de la Genèse. Les colonnes de I à XVII concernent Noé, depuis sa naissance jusqu'au partage de la terre entre ses enfants. Étant donné le caractère fragmentaire de ces colonnes (notamment la fin de la col. XVII et la col. XVIII), il est impossible de déterminer l'intégralité de la trame narrative. Néanmoins, on peut distinguer certains épisodes de la biographie noachique et surtout l'emploi de la première personne, ce qui transforme une bonne partie de ce texte en une suite autobiographique de Noé et d'Abraham. Ce trait est

19. Ursula Schattner Rieser, «Histoire des Patriarches », dans K. Berthelot, Th. Legrand, A. Paul (éds), La Bibliothèque de Qumrân, tome 1 Torah. Genèse, Paris, Cerf, 2008, pp. 319-387. Sauf indication contraire, nous suivons cette traduction. Voir aussi M. J. Bernstein, « From the Watchers to the Flood : Story and Exegesis in the Early Columns of the Genesis Apocryphon », dans E. G. Chazon, D. Dimant, R. A. Clements (éds), Reworking the Bible: Apocryphal and Related Texts at Qumran. Proceedings of a Joint Symposium by the Orion Center for the Study of the Dead Sea Scrolls and Associated Literature and the Hebrew University Institute for Advanced Studies Research Group on Qumran, 15-17 January, 2002, Leiden, Brill, 2005 ; Moshe J. Bernstein, «Re-arrangment, Anticipation and Harmonization as Exegetical Features in the Genesis Apocryphon », Dead Sea Discoveries, 3:1, 1996, pp. 37-57 ; Daniel J. Harrington, « The Bible Rewritten (Narrative) », dans R. A. Kraft, G. W. E. Nickelsburg (éds), Early Judaism and its Modern Interpreters, Atlanta, Scholars Press, 1986, pp. 239-247. 
remarquable car très rare dans la littérature de cette époque et, d'un point de vue littéraire, inscrit cette création aux antipodes du livre de la Genèse où Noé ne prend jamais la parole.

L'Apocryphe de la Genèse partage certains thèmes avec 1 Hénoch et d'autres avec Jubilés, sans pour autant qu'on soit en mesure d'établir clairement une relation d'emprunt. Ainsi donc, la naissance de Noé rappelle l'épisode similaire décrit dans 1 Hénoch (voir supra) : l'inquiétude de Lamech quant à sa paternité, la quête de Mathusalem auprès de son père Hénoch pour trouver réponse à cette interrogation, la prophétie rassurante de celui-ci au sujet de l'enfant Noé (1QapGen V). Entre les nombreuses lacunes du manuscrit, on peut lire une ligne mentionnant en quoi ce nouveau-né sortait du commun : «il leva les yeux sur moi et ses yeux brillèrent comme le sol[eil] $»^{20}$. La compréhension de ce passage dépend directement des parallèles dressés avec 1 Hénoch, sans lesquels les bribes de textes conservées ne nous livreraient pas un sens cohérent.

Comme dans Jubilés, Noé est crédité d'avoir transmis à la postérité un écrit - ktav miley Noah (1QapGen V, 29) - dont le contenu nous est livré, à la première personne, à partir de la colonne VI. Il ne s'agit pas ici de remèdes, ni d'instructions cultuelles, mais d'une quasi-autobiographie. Noé commence par interpréter sa propre naissance, se montrant tout à fait conscient de son destin, celui de garant de « la vérité » (qshot) :

Loin de l'injustice et dans le creuset de celle qui fut enceinte de moi, je suis apparu pour la vérité ; et lorsque je suis sorti des entrailles de ma mère, (c'est) pour la vérité que j'ai été planté. Et tous mes jours, je les ai conduits dans la vérité, et j'ai marché dans les chemins de l'éternelle véritéé ${ }^{2}$.

Noé poursuit son autobiographie en nous faisant part d'une première vision, associant ainsi son image à celle de son aïeul Hénoch, dans le livre éponyme. Cette vision informe le héros sur l'origine du mal :

Dans une vision je vis et /je/ fus informé et renseigné au sujet des activités des fils du ciel et comment tout [...] des cieux. Alors j'enfouis ce mystère dans mon cœur et ne le fis connaître à personne. (VI.11-12)

20. Apud Schattner-Rieser ibidem, sur la base du ms 1Q19 35.

21. Voir aussi col. VI lignes 3, 4, 6, 23. 
Malheureusement, les événements qui précèdent le déluge et le déluge lui-même ne peuvent pas être reconstitués dans leurs spécificités mais uniquement en prenant comme support les versions mieux conservées des passages équivalents dans Jubilés et 1 Hénoch. Ces lacunes concernent également le sacrifice offert à la sortie de l'arche - «je fis une expiation pour la terre tout entière. » (1QapGen VIII.6) - dont seulement certains éléments ont subsisté : « le bouc en premier », « en troisième lieu les jeunes tourterelles », «dessus je mis la farine mélangée à de l'huile, avec l'encens en oblation», «sur chaque offrande je mis du sel $»^{22}$. Élément narratif inédit, à la sortie de l'arche Noé prend symboliquement possession de la terre en la parcourant «en long et en large $»^{23}$, une terre «couverte d'herbe, de verdure et de grains », tous signes visibles de son renouvellement (XI.12). La stature de Noé en tant qu'interlocuteur direct de Dieu est renforcée par une deuxième vision énonçant le régime alimentaire des humains, régime végétarien accompagné d'un régime carné dont le sang est banni. Les colonnes XIII-XV nous présentent un songe symbolique de Noé dont il faut noter surtout la similitude avec le songe d'Abram (XIX.14-16) et dont, une fois de plus, le caractère fragmentaire empêche la compréhension.

De cette énumération nous retenons surtout le rapprochement récurrent entre Noé et Abram, ce qui inscrit l'Apocryphe de la Genèse dans le système de pensée ayant inspiré le livre des Jubilés. Ici aussi les techniques de composition sont utilisées pour exprimer l'idée que Noé anticipe Abram non seulement chronologiquement mais typologiquement.

\section{Conclusions}

Les textes que nous venons d'étudier sont d'une grande diversité dans la manière de concevoir le personnage de Noé. D'une part, il varie en intensité : tantôt effacé ${ }^{24}$ voire anonyme, tantôt figure

22. Notons l'absence du vin.

23. Comme Abraham en 1QapGen XXI.14.

24. Dans au moins un texte, 2 Hénoch ou Hénoch slave, toutes les fonctions dont Noé est généralement crédité sont attribuées à son frère cadet, Nir. Ce fait est de nature à confirmer l'hypothèse d'une variation textuelle de type mythologique 
prééminente consciente de sa propre valeur. D'autre part, ses attributs varient considérablement : figure quasi-messianique, sacerdotale ou prophétique, poseur de limites, législateur ou exorciste. Le trait d'union entre ces différents aspects est son caractère axial : Noé incarne l'élément de continuité entre l'ancienne et la nouvelle humanité, entre un premier monde et sa refondation après le déluge. Il remplit sa fonction de manière passive, de par sa simple élection, ou active, de par son action réparatrice et ordonnatrice.

La fonction de Noé est également liée à la manière dont l'humanité est censée faire face au mal. Il est tout à fait remarquable que dans les différents textes cités, l'origine du mal n'est pas humaine ou du moins, selon les variantes, pas directement humaine. Le mal est l'œuvre des êtres célestes, dont la nature divine demeure toujours un questionnement d'ordre théologique mais dont la nature nonhumaine ne fait pas débat. Dans ce système de pensée, le mal est une nécessité, inéluctable au monde. Dans la Bible, un verset d'Isaïe laisse entrevoir cette même conception - «Je forme la lumière et je crée les ténèbres, je donne la paix et je crée le mal ( $\left.\mathrm{ra}^{6}\right)$ » (Is 45.7) - tout comme le mauvais esprit qui vient du Seigneur pour tourmenter Saül (1 Samuel). On retrouvera la même idée dans la philosophie rabbinique du mal par le biais de quelques aggadot. Ainsi, on explique la raison d'être de la folie et, par extension, de toute forme de vie maléfique, par sa fonction paradoxalement protectrice, comme ce fut le cas de David qui, pour échapper au roi philistin de Gath, avait feint la folie. Tout une tradition existe dans le judaïsme qui attribue le mal non pas au libre arbitre de l'homme mais à des forces supérieures. Notons, cependant, un point commun entre ces deux philosophies selon lesquelles le mal est double: sexuel et gnoséologique. Dans les deux cas l'homme pêche par ce qu'il découvre le sexe et acquiert la connaissance.

Dans ce scénario, Noé joue parfois le rôle non seulement de survivant et continuateur de l'humanité première, mais aussi de réparateur du mal. Il est décrit dans certains passages sous des traits sacerdotaux et, dans ce cas, c'est par le sacrifice qu'il rachète le mal. Dans d'autres passages, il est le dépositaire de formules magiques

du personnage de Noé, plutôt que celle d'une réécriture biblique. V. Andrei Orlov, "Noah's Younger Brother': The Anti-Noachic Polemics in 2 Enoch", Henoch, no. 22, 2000, pp. 207-221. 
pour éloigner les démons et de remèdes pour guérir la maladie. Savoir ritualiste d'un côté, savoir magique de l'autre.

Cette théodicée correspond bien à ce que nous connaissons de la théologie du Yahad à Qumrân et notamment du déterminisme anthropologique et historique. 1 Hénoch, Jubilés, Apocryphe de la Genèse font tous partie de l'ensemble documentaire découvert dans les grottes du désert de Judée. Chronologiquement, il est fort probable que leur composition précède l'installation du groupe de Qumrân et, par conséquent, si on peut les qualifier de textes communautaires ce n'est pas en vertu de leur rédaction dans ce scriptorium mais en vertu de leur accréditation par l'usage. Le dualisme qumrânien considère que le bien et le mal régissent ensemble l'individu et le monde, selon un subtil et instable équilibre. De même, l'insistance sur l'aspect messianique dans 1 Hénoch explique peut-être l'attestation de cet écrit à Qumrân. La corruption de la terre à la fin des temps et à la veille du déluge correspond bien à la description de la situation actuelle telle qu'elle se reflète par exemple, dans la Règle de la Communauté. Le Yahad interprète le moment historique contemporain comme annonçant la fin des temps et s'identifie au reste survivant. Cependant il ne s'agit pas de n'importe quel reste mais de celui qui conclura une nouvelle alliance et acceptera une nouvelle alliance à la manière de Moïse et d'Abraham. Pour cette raison, les textes qui opèrent ce télescopage entre alliance noachique et alliance mosaïque sont particulièrement valorisés puisque de cette manière le particularisme juif est préfiguré dans l'universel noachique. Telle est, à nos yeux, la principale leçon de l'usage que les textes du Second Temple font de la figure de Noé. 